\title{
Varicella Zoster Virus Anterior Uveitis Complicated by Thalamic Stroke
}

\section{Casey Anthony}

Emory University

Anastasios P. Costarides

Emory Eye Center

Steven Yeh

Emory Eye Center

Jessica G. Shantha ( $\boldsymbol{D}$ jshanth@emory.edu )

Department of Ophthalmology, Emory Eye Center

\section{Brief report}

Keywords: infection, inflammation, virus, ophthalmic

Posted Date: August 28th, 2020

DOl: https://doi.org/10.21203/rs.3.rs-65407/v1

License: (9) This work is licensed under a Creative Commons Attribution 4.0 International License.

Read Full License

Version of Record: A version of this preprint was published at Journal of Ophthalmic Inflammation and Infection on May 1st, 2021. See the published version at https://doi.org/10.1186/s12348-021-00243-5. 


\section{Abstract}

We report a case of varicella zoster virus (VZV)-associated anterior uveitis in a patient with weight loss, arthritis and signs of inflammatory bowel disease. Her clinical course included the development of a thalamic stroke secondary to VZV cerebral vasculopathy. Following antiviral therapy, the patient's neurologic symptoms recovered and her ophthalmic findings improved.

\section{Case Report}

A 30-year-old woman with a history of a 20-pound weight loss within one year, gastrointestinal symptoms suggestive of inflammatory bowel disease, and an inflammatory arthritis had been started on an oral prednisone taper two months prior to presentation to our service. Two weeks after starting prednisone, she developed progressive pain and decreased vision in the right eye. She was diagnosed with a unilateral anterior uveitis, which was initially deemed to be associated with her systemic inflammatory condition by an outside provider. The patient was started on topical prednisolone acetate $1 \%$ drops four times daily, with only mild improvement in her symptoms. She was then advanced to difluprednate (Durezol) four times daily, given its increased corticosteroid potency. Because of increasing eye pain and inflammation, she was referred to our service for management.

The patient's presenting Snellen visual acuity was 20/40 in the right eye (OD) and 20/20 in the left eye (OS). The pupils were $9 \mathrm{~mm}$ and akinetic OD and $5 \mathrm{~mm}$ with brisk light reactivity OS. Her intraocular pressure (IOP) was extremely elevated at $65 \mathrm{mmHg}$ OD (Normal 9-22 $\mathrm{mmHg}$ ) and $21 \mathrm{mmHg}$ OS. Slit lamp examination showed pigmented, granulomatous keratic precipitates (KP) within the inferior cornea, $3+$ anterior chamber cell, diffuse iris atrophy with pigment on the zonular fibers of the lens, and ectropion uveae (Fig. 1). Gonioscopy showed $3+$ pigmented cells within the inferior angle OD. Dilated fundus exam was unremarkable in both eyes. At the time of presentation, she had been off oral prednisone for 3 weeks and off topical corticosteroid drops for 3 days.

A diagnostic anterior chamber paracentesis to assess the aqueous humor was positive for VZV DNA by PCR testing and negative for HSV, CMV, and toxoplasmosis DNA. Serologic testing showed positive VZV IgG antibody. She was diagnosed with VZV-associated hypertensive anterior uveitis, prompting the initiation of oral acetazolamide and ophthalmic timolol, dorzolamide, and brimonidine for ocular hypertension, as well as valacyclovir $1 \mathrm{~g}$ TID and prednisolone acetate $1 \%$ every 2 hours to treat the VZVassociated anterior uveitis.

Two days after starting valacyclovir, the patient developed left-sided lip, arm, and leg numbness and tingling and was hospitalized for a cerebrovascular accident. MRI showed a right-sided thalamic lacunar infarct (Fig. 1). The thalamic stroke was thought to be due to VZV-associated CNS vasculopathy, and she was empirically started on two weeks of IV acyclovir and aspirin with resolution of the majority of her CNS symptoms with the exception of a residual minor left-sided weakness. She resumed oral valacyclovir ( $1 \mathrm{~g}$ TID) and topical corticosteroid after her treatment course with IV acyclovir. The patient's eye pain 
initially improved and her visual acuity remained stable with an IOP of $13 \mathrm{mmHg}$ OD two weeks after her hospitalization.

However, two months after her stroke, she presented with a recurrence of pain and blurred vision OD. The patient's visual acuity had declined to 20/100 and her IOP was elevated at $39 \mathrm{mmHg}$. Anterior segment exam showed $1+$ ciliary injection and $3+$ anterior chamber cell. The differential diagnosis for her recurrent ocular symptoms and findings included valacyclovir resistance or hypertensive anterior uveitis due to zoster recurrence. Her elevated IOP was thought to be due to either a corticosteroid response, secondary open angle glaucoma due to dense pigmentary obstruction of her trabecular meshwork, acute trabeculitis, or a combination of these factors. She was switched from prednisolone to difluprednate with minimal improvement in the intraocular inflammation. Because of her persistently elevated IOP despite maximum medical ocular hypertensive therapy, she underwent a glaucoma surgery with an Ahmed tube shunt with a postoperative improvement of her IOP to $11 \mathrm{mmHg}$. The patient's ocular inflammation eventually resolved while she remained on valacyclovir (1 g TID) and topical prednisolone acetate $1 \%$ QID.

At 6-month follow-up, the patient's symptoms and exam findings improved with a visual acuity of 20/80 and IOP of $15 \mathrm{mmHg}$, but she continued to exhibit 1 + pigmented cell. At final follow-up 10 months following her initial evaluation, her exam stabilized with visual acuity of 20/50 and only rare anterior chamber pigment cells (Figure). The patient has continued valacyclovir (1 g TID), prednisolone acetate $1 \%$ TID, and timolol BID. She was also diagnosed with early Crohn's disease during her clinical course, but no additional systemic immunosuppressive medications were recommended the time given the risk of exacerbating the viral disease process.

\section{Discussion}

Anterior uveitis can be associated with infectious or noninfectious etiologies, and timely diagnosis is essential to guide appropriate treatment and minimize the potential for systemic morbidity, particularly in cases where non-ophthalmic findings develop $[3,4]$. Our patient was undergoing workup for inflammatory bowel disease and arthritis and was treated initially with oral corticosteroids, which may have complicated her disease course. Anterior uveitis is deemed to be idiopathic in approximately $50 \%$ of cases and often does not require extensive workup, particularly for the first episode of anterior uveitis; however, a viral etiology (i.e. varicella zoster, herpes simplex, cytomegalovirus, or rubella) should be strongly considered in the differential diagnosis when uveitis is accompanied by ocular hypertension or iris atrophy, both of which were observed in our patient's case [2].

VZV-associated anterior uveitis may be mild and self-limiting; however, in some cases, chronic or recurrent disease may develop. Complications can include iris atrophy, pupillary abnormalities (i.e. posterior synechiae, pupillary dysmotility from sphincter dysfunction and atrophy), acute IOP elevations, and glaucomatous optic neuropathy. Interestingly, our patient did not show typical cutaneous manifestations and presented at a younger age than most individuals, as VZV anterior uveitis is more 
commonly observed in elderly patients. Even in the absence of a history of herpetic zoster ophthalmicus, clinical suspicion for a viral process should remain high in patients with characteristic ophthalmic findings [3]. PCR of aqueous humor is a highly sensitive test to confirm the diagnosis following aspiration of intraocular fluid, which can be performed in the ophthalmic clinic setting [8].

After presenting with anterior uveitis, the patient developed a stroke, which was consistent with CNS vasculopathy previously described with VZV. Prior studies show an up to 10 -fold increased stroke risk in patients with VZV as a result of viral infection in the arterial wall of cerebral vasculature. VZV vasculopathy was historically associated with large-vessel stroke and acute hemiplegia, but the clinical spectrum of VZV vasculopathies can include transient ischemic attacks, ischemic and hemorrhagic stroke involving both small and large vessels, aneurysm, cranial neuropathies, and venous sinus thrombosis. While VZV vasculopathy is treatable, this diagnosis is often missed and no antiviral treatment is administered, as one-third of patients have no documented history of a zosteriform rash, and many patients may have normal CSF findings $[1,6,7,9]$.

The differential diagnosis of the combination of anterior uveitis and stroke includes both infectious and noninfectious conditions. These include viral etiologies (VZV, HSV), tuberculosis, syphilis, sarcoidosis, granulomatous polyangiitis, and neuro-Behcet's disease. Systemic lupus erythematosus and rheumatoid arthritis are less commonly associated with anterior uveitis, and more often associated with scleritis as their predominant ocular inflammatory disease finding, but still warrant consideration in patients with typical systemic findings. Our patient's treatment of her stroke included empiric intravenous acyclovir by the treating facility given her recent uveitis diagnosis. Ultimately, the patient's neurologic symptoms and disease findings abated.

The initial diagnosis of VZV-associated hypertensive anterior uveitis, diffuse iris atrophy with pigment release, and disease course was atypical in the patient's age, degree of pigment change, and the potentially debilitating stroke. Few cases have been reported of a stroke following diagnosis of ocular VZV [5]. However, recognition of the relationship between the ophthalmic and CNS manifestations was important for delivery of multidisciplinary care. In addition, this case emphasizes the importance of differentiating between infectious and noninfectious etiologies of uveitis, as there were additional concerns about VZV persistence and potential disease recurrence in the eye with corticosteroid and other immunosuppressive medications. Besides the potential of exacerbating anterior uveitis with immunosuppression, individuals with viral anterior uveitis may also develop sight-threatening retinitis (i.e. acute retinal necrosis) following immunosuppression. The recognition of ocular VZV was instrumental for prompt antiviral therapy, identifying the underlying cause of stroke, and avoiding potential morbidity from immunosuppressive medications.

\section{Abbreviations}

BID: two times per day; CNS: central nervous system; HSV: herpes simplex virus; IOP: intraocular pressure; MRI: magnetic resonance imaging; OD: right eye; OS: left eye; PCR: polymerase chain reaction; QID: four 


\section{Declarations}

\section{Acknowledgements}

Not applicable

\section{Competing interests}

The authors declare they have no competing interests.

\section{Availability of data and materials}

Data sharing is not applicable to this article as no datasets were generated or analyzed during the current study.

\section{Ethics Approval and consent for publication}

Ethics approval was not applicable and consent to publish were waived as no personal identifiable information is disclosed

\section{Authors' contributions}

CA analyzed the data, drafted and revised the manuscript. AC gathered the data, participated in data analysis, edited and approved the final manuscript. JS and SY gathered and analyzed the data, drafted, revised, and approved the final manuscript.

\section{Funding}

This project was supported by the National Eye Institute/ National Institutes of Health core grant P30EY06360 (Department of Ophthalmology, Emory University School of Medicine), National Eye Institute of the National Institutes of Health under award number K23 EY030158 (Shantha) and R01 EY029594 (Yeh). The content is solely the responsibility of the authors and does not necessarily represent the official views of the National Institutes of Health or the views or policies of the Department of Health and Human Services, nor does mention of trade names, commercial products, or organizations imply endorsement by the U.S. Government. This research was also supported an unrestricted departmental grant from Research to Prevent Blindness, Inc. to the Emory Eye Center, Emory University School of Medicine and Association for Research in Vision and Ophthalmology Mallinckrodt Young Investigator Award (Yeh).

\section{References}


1. Amlie-Lefond C, Gilden D (2016) Varicella Zoster Virus: A Common Cause of Stroke in Children and Adults. J Stroke Cerebrovasc Dis 25:1561-1569

2. Chan NS-W, Chee S-P (2019) Demystifying viral anterior uveitis: A review. Clinical \& Experimental Ophthalmology 47:320-333

3. De-La-Torre A (2018) Virus-Induced Anterior Uveitis (VIAU) in Immunocompromised Patients. Ocular Immunology and Inflammation 26:807-817

4. Harthan JS, Opitz DL, Fromstein SR et al. (2016) Diagnosis and treatment of anterior uveitis: optometric management. Clin Optom (Auckl) 8:23-35

5. Hashemi N, Zhang J, Volpi J et al. (2013) A pox upon your house. Survey of ophthalmology 58:640643

6. Nagel MA, Cohrs RJ, Mahalingam R et al. (2008) The varicella zoster virus vasculopathies: clinical, CSF, imaging, and virologic features. Neurology 70:853-860

7. Nagel MA, Gilden D (2014) Update on varicella zoster virus vasculopathy. Curr Infect Dis Rep 16:407407

8. Tugal-Tutkun I, Cimino L, Akova YA (2018) Review for Disease of the Year: Varicella Zoster VirusInduced Anterior Uveitis. Ocular Immunology and Inflammation 26:171-177

9. Yawn BP, Wollan PC, Nagel MA et al. (2016) Risk of Stroke and Myocardial Infarction After Herpes Zoster in Older Adults in a US Community Population. Mayo Clin Proc 91:33-44

\section{Figures}


A

B
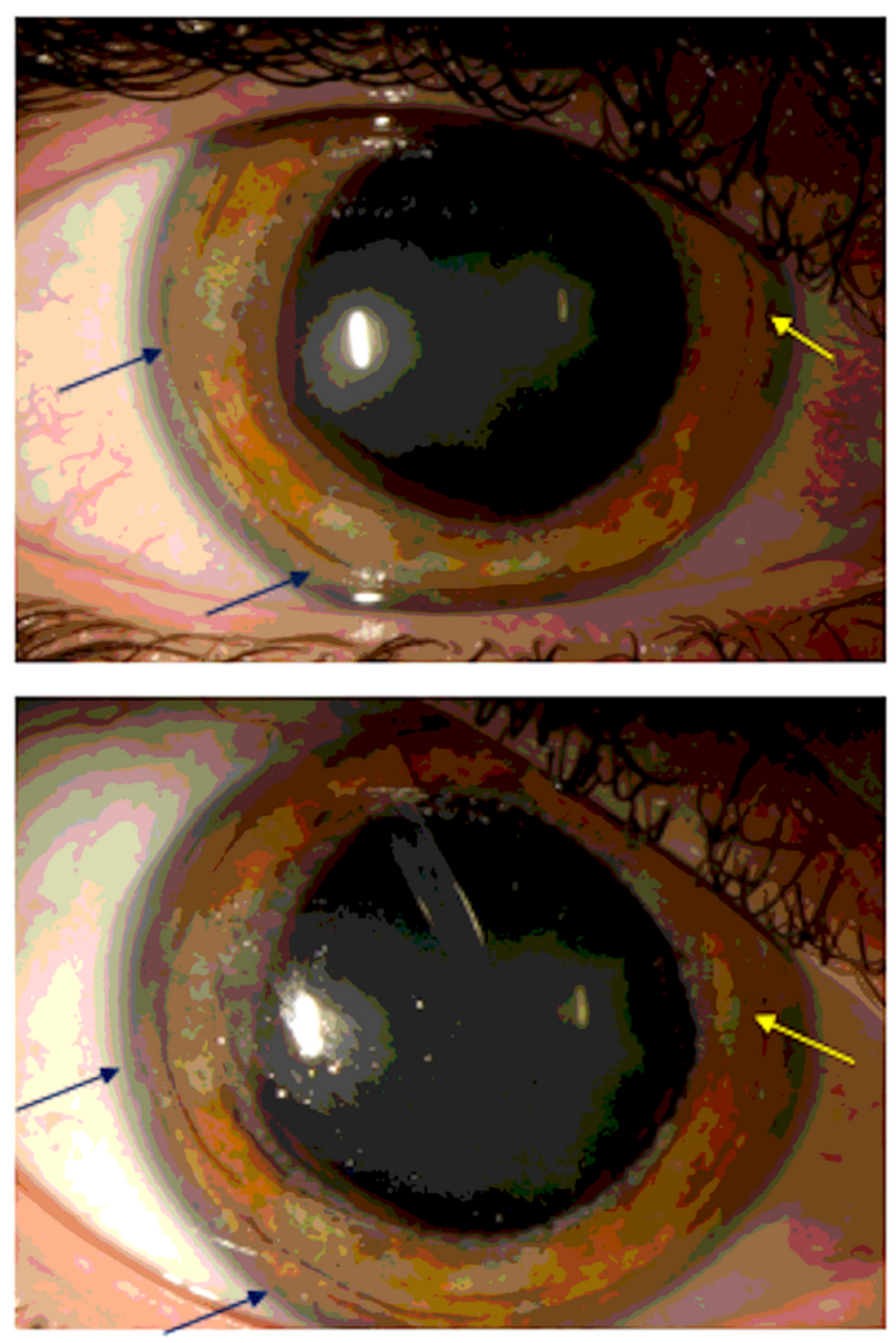

C

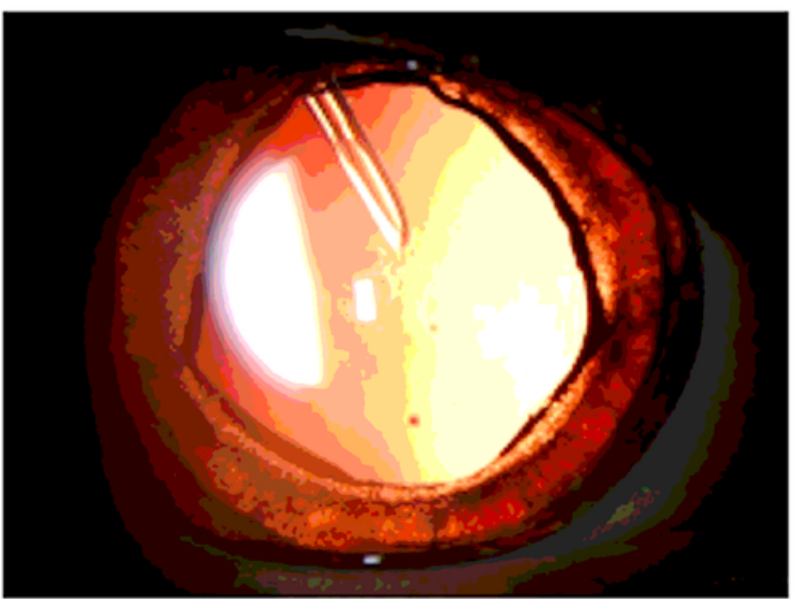

$\mathbf{D}$

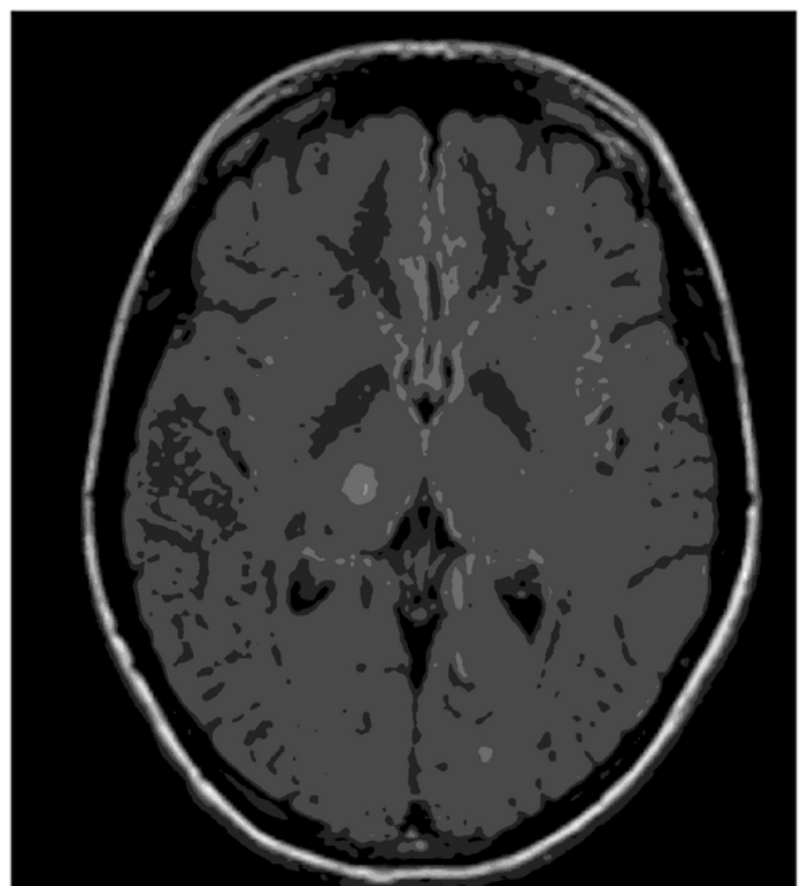

\section{Figure 1}

Slit-lamp photo showed multifocal patches of iris atrophy temporally (blue arrows) and nasally (yellow arrow) at presentation (A). The areas of atrophy enlarged with increased pigment deposition at follow-up (B). These areas are readily visualized as diffuse transillumination defects with retroillumination (i.e. orange/red reflex off the retinal surface) which is visualized through the atrophic iris stroma and epithelium (C). A T2 FLAIR magnetic resonance image after the patient's stroke showed lacunar infarcts within the right thalamus (D). 